\title{
Centralized network intelligence vs. decentralized network intelligence
}

\author{
Josef Ferchenbauer \\ Siemens $A G$ \\ E-mail: Josef.Ferchenbauer@icn.siemens.de
}

Key words: Network intelligence, Private line service, Permanent Virtual Connection, Soft Permanent Virtual Connection

Abstract: Often operators and vendors have to decide whether a new service shall be introduced into the network via centralized or decentralized service logic. This paper shows the points that have to be considered in such cases. It works out the advantages and disadvantages of a centralized and decentralized network intelligence approach on an example. As an example an existing service, the private line service offered via an ATM network, is taken. The example is used to picture and verify the results, but the results are universally applicable. In cases where they are not it is stated explicitly.

\section{INTRODUCTION}

This paper shows the points that have to be considered by operators and vendors to decide whether a service shall be introduced into the network via centralized or decentralized service logic.

The first part of the paper describes the service that has been taken as an example. The second part describes the advantages and disadvantages of a centralized and decentralized approach using the example.

\footnotetext{
The original version of this chapter was revised: The copyright line was incorrect. This has been
} corrected. The Erratum to this chapter is available at DOI: 10.1007/978-0-387-35522-1_37 
2. PRIVATE LINE SERVICE

\subsection{Private Line Service via PVCs}

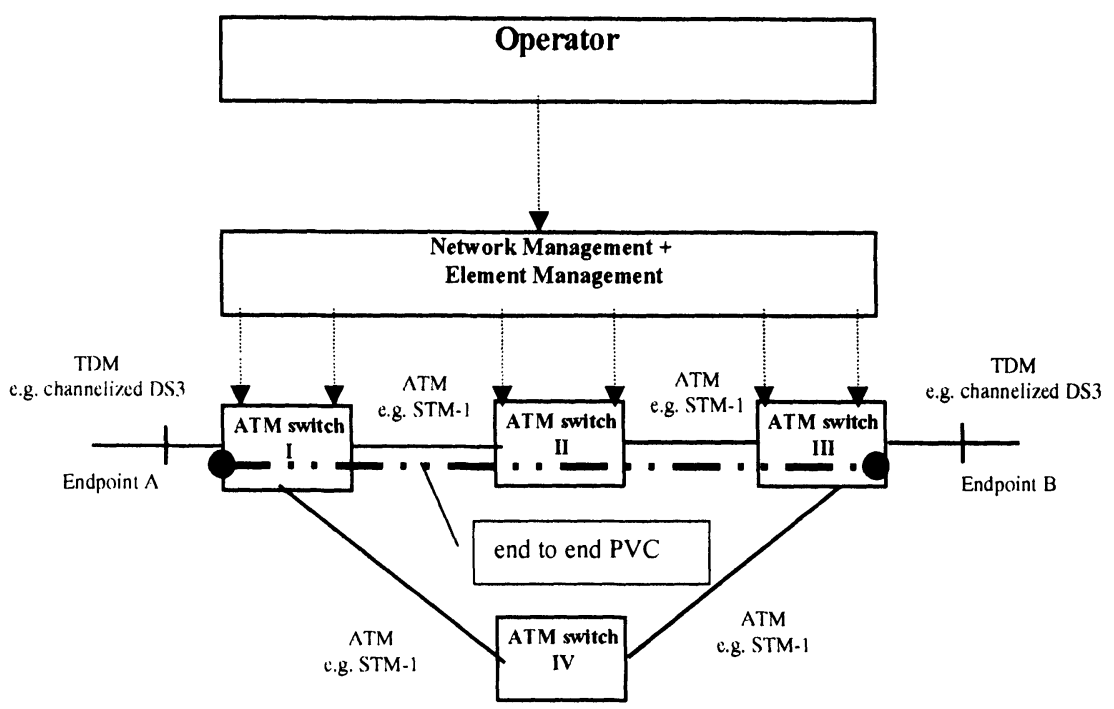

Figure I. Private line service offering via PVCs (centralized network intelligence)

A private line is a permanent connection between two endpoints, in the given example endpoint $\mathrm{A}$ and endpoint $\mathrm{B}$. The establishment of the permanent connection can be done by the operator via TMN, i.e. the operator initiates the through connection between Endpoint A and the ATM link in switch I. Then the operator connects both ATM links in switch II. Finally the through connection between the ATM link and Endpoint B has to be performed. Then a so-called PVC between Endpoint A and Endpoint B is established. To support the operator an intelligent application in the network management can do the routing, therefore the operator has to define only Endpoint $\mathrm{A}$ and Endpoint $\mathrm{B}$. The rest is performed by the network management itself. This application is called end-to-end PVC establishment. 


\section{Operator}

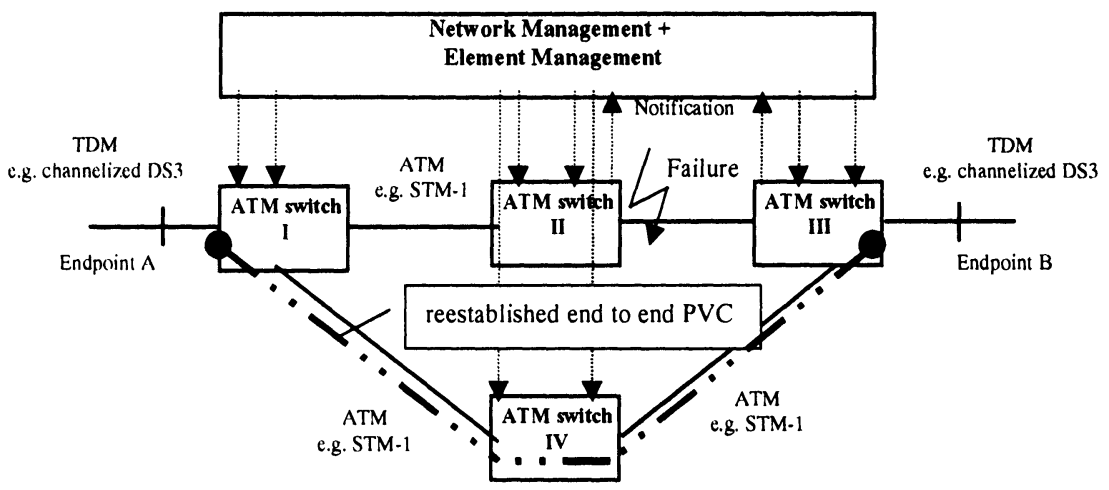

Figure 2. Private line reestablishment via PVCs

It is very important that these connections are highly reliable, i.e. the downtime of such connections has to be minimized as much as possible. Therefore in case of a link or node outage the network management is notified about the outage. This triggers the end-to-end PVC application in the network management to establish the connection via an alternative path, e.g. via switch IV instead of switch II.

End-to-end PVC establishment is an example for centralized network intelligence. 


\subsection{Private Line Service via SPVCs}

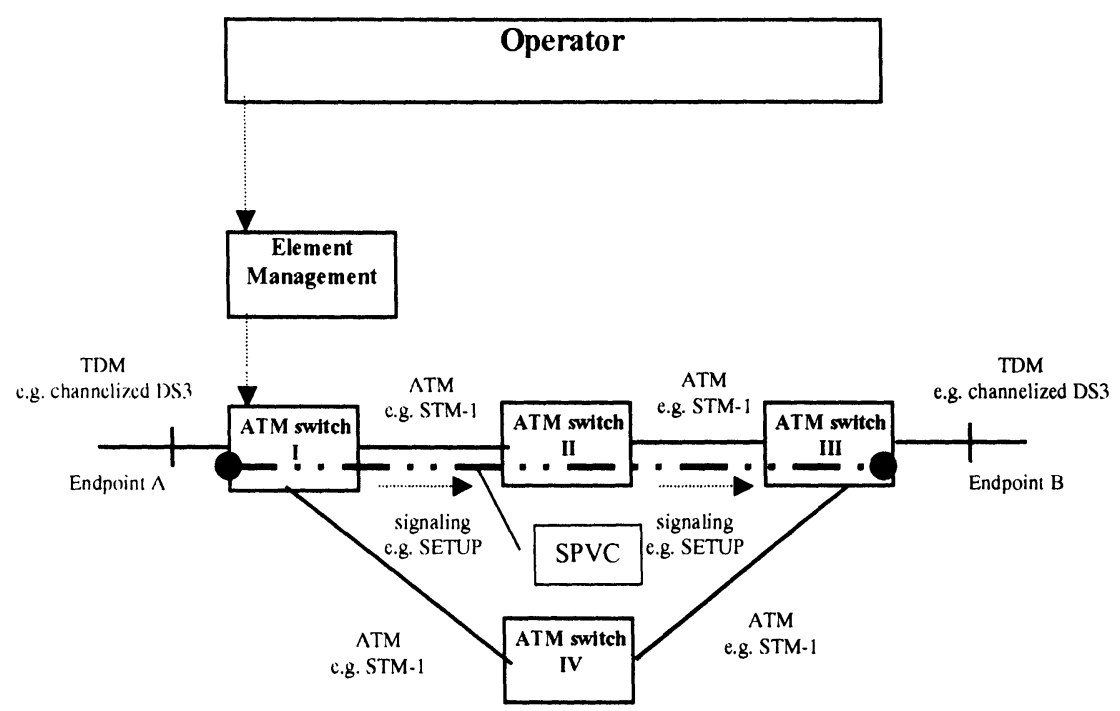

Figure 3. Private line service offering via SPVCs (decentralized network intelligence)

Another approach is to use soft Permanent Virtual Connections (SPVCs) to establish a private line between the endpoints. The operator defines Endpoint A (originating endpoint) and Endpoint $\mathrm{B}$ and sends the request to establish a connection between both endpoints to switch I. Endpoint B is identified by a unique address (called party number). Then switch I sets up a connection, using SVC functionality (signaling, routing etc.), towards endpoint B at switch III. This connection is a so-called Soft Permanent Connection (SPVC). Each switch itself has to perform routing and through connection, therefore this is an example for decentralized network intelligence. For this approach no network management is required; a simple element management is fully sufficient. 


\section{Operator}

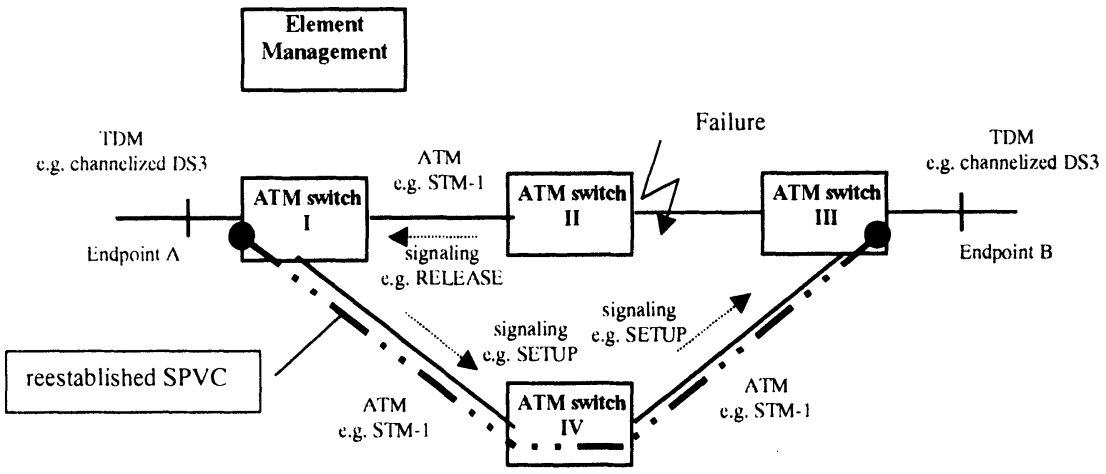

Figure 4. Private line reestablishment via SPVCs

The originating endpoint (Endpoint $A$ at switch I) is responsible for maintaining the end-to-end connection by setting up a new switched connection automatically in case the existing switched connection fails. This is to minimize the downtime of such connections, which is very important for this service

One approach of failure handling is to tear down the connection, i.e. the neighbor node of a faulty entity issues a release of the connection via signaling. When Switch I receives the release message it tries to find another path towards Endpoint B through the network. This reestablishment will result in a connection via switch IV instead of switch II.

All this is achieved without any centralized network intelligence, i.e. without a centralized network management. Therefore SPVCs are an example of decentralized network intelligence.

To support SPVCs in a multi vendor environment the signaling enhancements for this service has been standardized by ATM-Forum /1/ /2/ and ITU $/ 3 /$. 


\section{COMPARISON}

\subsection{Time to market}

The PVC (centralized) approach has the advantage that only one network element, the network management, has to be upgraded. A precondition for this is that the ATM switch already offers an interface to establish PVCs inside the switch and to notify the network management of faults. These are basic functions, therefore most of the ATM switches offer such functions.

In case of the SPVC (decentralized) approach each switch has to be upgraded to support SPVCs. In addition the switches have to support SVCs because this is the basic function used for SPVC establishment. In addition the signaling protocols to support SPVCs has to be standardized. For SPVCs this has already been done but for new services this might take some time and defer new features. For a short term solution signaling might be enhanced proprietary, but for multi vendor networks standards are crucial.

Assessment: As long as only already available basic functions of the switch are used to create a new service the centralized approach has clear advantages.

\subsection{Performance}

The establishment and reestablishment of PVCs has to be performed by network management. Only one PVC after the other can be established respectively reestablished. Thus network management becomes a bottleneck especially in case of failure. When a failure occurs the network management has to search a new path through the network and create the new connection. This reestablishment is very time critical because the interruption of the connection, due to a failure, has to be very short.

SPVC are established and reestablished from the originating endpoint. Only the nodes, which are carrying the SPVC, are involved in establishing and reestablishing. The establishment and reestablishment of SPVCs will be done in parallel. Each switch only processes its SPVCs.

Assessment: From the performance point of view the decentralized solution has clear advantages. This is because the functions (in our example establishment and reestablishment) are performed in parallel. 


\subsection{Scalability}

As the network grows the processing power which is usable by the service logic grows with it in case of the decentralized service support. In case of the centralized approach it does not.

Assessment: The decentralized approach has its advantages, but this is not a point in its own rights because it is already covered by the performance investigation.

\subsection{Reliability}

Central approach: In case that the central entity which hosts the service logic, in our example the network management, fails the service cannot be supported anymore. For instance no reestablishment can be performed.

Distributed approach: In this case when the entity, which hosts the service logic, in our example, an ATM switch fails, only a fraction of the service is influenced by the failure. In addition when the switch fails it has the same influence on the central approach. In our example when the switch which has the endpoints connected (switch I and switch III) fails no establishment or reestablishment can be performed. This is valid for both approaches.

Assessment: The influence of hardware failures of the controller that host the service logic is much smaller in the decentralized approach.

\subsection{Self Organizing Network Support}

In self-organizing networks, e.g. PNNI networks, there is no central entity required that has the knowledge of the whole network. But for certain services like the private line service this is necessary when the centralized approach is chosen.

Assessment: The decentralized approach does fit best into a plug and play environment. By softening the plug and play philosophy, e.g. by configuring the physical nodes, physical links and user addresses via a central management entity, also the centralized approach can be used. But then the question is whether it is still a plug and play network?

\subsection{Multi Vendor Ability}

Central approach: Network management has to be able to support the different management interfaces of the switches, but the interface can be 
negotiated between the network management vendor and each switch vendor separately.

Distributed approach: The interface has to be negotiated between all vendors of the network. A practical way to achieve this is to standardize the feature if it shall be supported in a multi vendor environment.

Assessment: Both approaches can be used in multi vendor networks. The distributed approach needs more coordination effort, but this becomes a time to market issue in the end.

\subsection{Operational Costs}

The operational cost for both approaches is the same, because the operator has to put in the endpoints and maybe some ATM traffic parameters for both solutions.

In case of SPVCs the signaling and routing infrastructure might have to be configured. This is only valid for networks where no SVC service is offered. If the SVC service is offered anyway the same signaling and routing infrastructure can be used.

Assessment: In certain cases (see above) the operational costs for the centralized solution are less.

\subsection{Cost of Purchase}

This point can only be answered on a case by case base. The only thing, which can be stated, is that a centralized solution needs a network management entity, which causes additional hardware costs.

\subsection{Service limited to network}

The end to end PVC management application, which is running on the network management entity, can only establish PVC via switches that are managed by it.

An SPVC need not to be limited to a network. This is because the terminating endpoint, i.e. Endpoint B in the example above, is identified by a unique address. The only precondition is that the other network supports the according standard $/ 1 / / 2 / 3 /$.

Assessment: This point is very special for this service; therefore it cannot be taken to derive a general statement for a centralized or decentralized approach. 


\subsection{Summary}

The following table summarizes the above made assessments.

\begin{tabular}{l|l|l} 
& $\begin{array}{l}\text { Centralized Network } \\
\text { Intelligence }\end{array}$ & $\begin{array}{l}\text { Decentralized Network } \\
\text { Intelligence }\end{array}$ \\
\hline Time to market & + & \\
\hline Performance & & + \\
\hline Scalability & & + \\
\hline Reliability & & + \\
\hline $\begin{array}{l}\text { Self organizing } \\
\text { network support }\end{array}$ & no & yes \\
\hline Multi vendor ability & $*$ & \\
\hline Operational cost & $*$ & \\
\hline Cost of purchase & & $*$ \\
\hline $\begin{array}{l}\text { Service limited to } \\
\text { network }\end{array}$ & yes & no
\end{tabular}

Table 1. Summary

Legend:

$+\ldots$ strength of the solution

* ... slightly better than the other solution

\section{CONCLUSIONS}

In this paper the advantages and disadvantages of a centralized and decentralized network intelligence approach have been investigated. This has been done for a specific service, the private line service. This does not mean that the result is only valid for this specific service, in the contrary the results are universally applicable.

The main advantage of a centralized approach is that services can be introduced in a network very fast (time to market). Therefore if a new service is introduced first on a small scale this approach is advantageous.

The main advantage of an decentralized approach is the performance and its scalability, i.e. when a service is commonly used in a network it has to be decentralized else the performance limits will be reached quite soon. 


\section{REFERENCES}

/1/ ATM Forum Technical Committee: Private Network-Network Interface Specification Version 1.0 (PNNI 1.0); af-pnni-0055.000; March 1996

12/ ATM Forum Technical Committee: ATM Inter-Network Interface (AINI) Specification AF-CS-0125.000; July, 1999

/3/ International Telecommunication Union:

ITU-T Recommendation Q.2767.1; Soft PVC capability

6. ABBREVIATIONS

PVC

SPVC

SVC

TMN
Permanent Virtual Connection Soft Permanent Virtual Connection Switched Virtual Connection Telecommunications Management Network 